Pacific Journal of Mathematics

SEMI-PRIMARY SPLIT RINGS 


\section{SEMI-PRIMARY SPLIT RINGS}

\section{GARRY HELZER}

This paper is concerned with Hochschild's "maximal algebra" which has also been discussed by Jans, Jans and Nakayama, and Zaks.

A category of rings for which the maximal algebra construction is valid is first defined. These are the "split rings" of the title. These rings include the split rings introduced by Jans and Nakayama but they need not be semi-primary. A second category consisting of a kind of sheaf over a directed graph is introduced. Using this second category the maximal algebra construction is exhibited as a composition of adjoint functors, and hence gives the universal mapping property of the maximal algebra. The properties of the sheaf category are then used to show that a semi-primary split ring is the image of a semi-primary ring of a special type.

In [6] the spectrum of a right perfect ring was modified to a rected graph. Although the resulting functor (rings) ${ }^{o p} \rightarrow$ graphs preserves and reflects coproducts it does not possess an adjoint. We modify the graph to a kind of sheaf by putting stalks of rings over the vertices and stalks of bimodules over the arrows. The resulting ring $\rightarrow$ (sheaves) $^{o p}$ has a right adjoint (sheaves) ${ }^{o p} \rightarrow$ rings and the composition functor rings $\rightarrow$ rings is essentially Hochschid's "maximal algebra" ([7], [8] and [9]).

Coupling this with known results (Theorem 2) gives the universal mapping property of the maximal ring of a split semi-primary ring [9].

Lastly we use the geometry of the sheaf category to show that that every semi-primary split ring is a homomorphic image of a certain type of subring of a split, hereditary generalized triangular matrix ring. This result is stated twice below. First, as Theorem 3 , it is stated in the special terminology of the sheaf category. Following Theorem 3 we make such definitions as are necessary to restate the result in ring theoretic terms: Theorem 3 '.

The formality of the proofs somewhat obscures the construction of the special ring so the construction is indicated for a special case following Theorem $3^{\prime}$.

1. Definitions. In this section we define the categories and functors to be used in subsequent sections. The symbols introduced will retain their meaning throughout the paper. 
By a "ring homomorphism" we understand an additive and multiplicative mapping of rings with unit which carries unit to unit. Unidentified maps are the obvious ones.

Definition. A split ring $R$ is a quadruple $(a, b, c, d)$ such that (R1) and (R2) below hold.

(R1) $a: R \rightarrow \Gamma, b: \Gamma \rightarrow R$ are ring homomorphisms with $a b=1_{\Gamma}$ and $\Gamma$ a semi-simple ring (with minimum condition).

If we set $I=\operatorname{ker} a$ then $I$ and $I^{2}$ are two-sided ideals of $R$ and hence $R-R$ bimodules. The map $b$ then induces a $\Gamma-\Gamma$ bimodule structure on $I$ and $I^{2}$.

(R2) $\quad c: I \rightarrow I / I^{2}, d: I / I^{2} \rightarrow I$ are $\Gamma-\Gamma$ bimodule homomorphisms such that $c d=1_{L}$.

We set $A=\operatorname{Im} d$ and then we have $R=\Gamma \oplus A \oplus I^{2}, I=A \oplus I^{2}$ as $\Gamma-\Gamma$ bimodules. This is the Jans-Nakayama definition (see [9]) when $I$ is the Jacobson radical of $R$ and $R$ is semi-primary.

Definition. A morphism of split rings $R \rightarrow R^{\prime}$ is a quadruple $(\alpha, \beta, \gamma, \delta)$ satisfying (R3), (R4), and (R5) below.

(R3) $\alpha: R \rightarrow R^{\prime}$ and $\beta: \Gamma \rightarrow \Gamma^{\prime}$ are ring homomorphisms, $\gamma$ and $\delta$ are induced by $\alpha$, and the diagrams
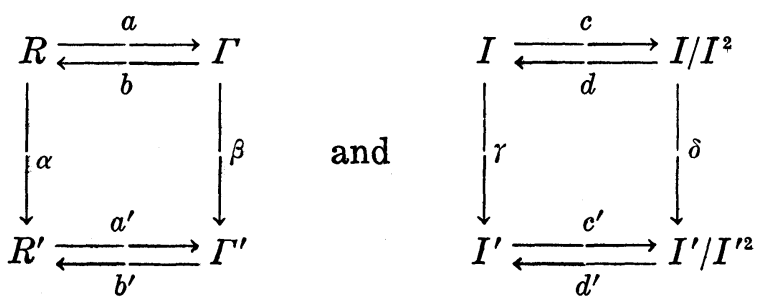

commute.

(R4) If $A^{\prime}$ is a maximal two-sided ideal of $\Gamma^{\prime}$ then

$$
\operatorname{ker}\left[\Gamma \longrightarrow \Gamma^{\prime} \longrightarrow \Gamma^{\prime} / A^{\prime}\right]
$$

is a maximal two-sided ideal of $\Gamma$.

(R5) If $A^{\prime}, B^{\prime}$ are maximal two-sided ideals of $\Gamma^{\prime}$ and

$$
\begin{array}{ll}
P^{\prime}=\operatorname{ker}\left[R^{\prime} \longrightarrow \Gamma^{\prime} \longrightarrow \Gamma^{\prime} / A^{\prime}\right], & Q^{\prime}=\operatorname{ker}\left[R^{\prime} \longrightarrow \Gamma^{\prime} \longrightarrow \Gamma^{\prime} / B^{\prime}\right] \\
P=\operatorname{ker}\left[R \longrightarrow R^{\prime} \longrightarrow R^{\prime} / P^{\prime}\right], & Q=\operatorname{ker}\left[R \longrightarrow R^{\prime} \longrightarrow R^{\prime} / Q^{\prime}\right]
\end{array}
$$

then the induced monomorphism $R / P Q \rightarrow R^{\prime} / P^{\prime} Q^{\prime}$ carries the Jacobson radical of $R / P Q$ onto the Jacobson radical of $R^{\prime} / P^{\prime} Q^{\prime}$.

By the split spectrum, $\operatorname{SSpec}(R)$, of $R$ we shall mean the set of ideals of $R$ of the form $\operatorname{ker}[R \rightarrow \Gamma \rightarrow \Gamma / A]$ as $A$ runs through the set of maximal two-sided ideals of $\Gamma$. The axiom (R4) then assures 
that a morphism $R \rightarrow R^{\prime}$ induces a map $\operatorname{SSpec}\left(R^{\prime}\right) \rightarrow \operatorname{SSpec}(R)$.

In [6] we introduced in a somewhat different context the skeleton, $S k_{r} R$, of a right perfect ring. Axiom (R5) assures that a morphism $R \rightarrow R^{\prime}$ induces a morphism $S k_{r} R^{\prime} \rightarrow S k_{r} R$. We shall denote the category of split rings by $S R n g$.

DEFINITION. A sheaf of simple rings over a finite graph, $F$, consists of

(S1) An oriented graph $G$. That is, a set of arrows $A_{G}$, a set of vertices $V_{G}$ and two functions $h, t: A_{G} \rightarrow V_{G}$ called head and tail. We assume that $A_{G}$ and $V_{G}$ are finite.

(S2) For each $x \in V_{G}$ a ring $R(x)$ which is simple (with d.c.c.).

(S3) For each $a \in A_{G}$ a left $R(t a)$ and right $R(h a)$ bimodule $M(t a, h a)$.

We further assume

(S4) The map $(t, h): A_{G} \rightarrow V_{G} \times V_{G}$ is one-to-one.

From now on we shall abbreviate "sheaf of simple rings over a finite graph" to "sheaf."

Definition. A morphism of sheaves $\eta: F \rightarrow F^{\prime}$ consists of

(S5) A morphism $\eta_{a}: G \rightarrow G^{\prime}$ of graphs. That is, a pair of functions $A_{G} \rightarrow A_{G^{\prime}}, V_{G} \rightarrow V_{G^{\prime}}$ which commute with $t$ and $h$.

(S6) For each $x \in V_{G}$ a ring homomorphism $\eta(x): R^{\prime}(\eta x) \rightarrow R(x)$.

(S7) For each $a \in A_{G}$ a homomorphism

$$
\eta(t a, h a): M\left(t^{\prime} \eta a, h^{\prime} \eta a\right) \longrightarrow M(t a, h a) \quad \text { of } \quad R^{\prime}\left(t^{\prime} \eta a\right)-R^{\prime}\left(h^{\prime} \eta a\right)
$$

bimodules. Here the bimodule structure on $M(t a, h a)$ is induced by $\eta(t a)$ and $\eta(h a)$.

We further assume

(S8) $\eta(t a, h a)$ is an isomorphism for each $a$ in $A_{G}$. We denote this category of sheaves by grass. Next we define a functor $S:(S R n g)^{o p} \rightarrow$ grass.

Let $R \in S R n g$. For the graph of $S R$ we adopt the skeleton of [6] to split rings. The vertices of the graph, $G$, are the elements of the set $\operatorname{SSpec}(R)$. If $P \in \operatorname{SSpec}(R)$ we set $R(P)=R / P$. We have an arrow $P \rightarrow Q \in A_{G}$ whenever the Jacobson radical of $R / P Q$ is nonzero. This radical is $P \cap Q / P Q$ which is a left $R / P$ and right $R / Q$ bimodule. If $P \rightarrow Q \in A_{G}$ we set $M(P, Q)=P \cap Q / P Q$. The axioms (R4) and (R5) now assure that the canonical definition of $S$ on morphisms defines a functor to grass.

Proposition 1. Let $R \in S R n g$ and give $R / I^{2}$ the canonical split ring structure. Then we may identify $S R$ and $S\left(R / I^{2}\right)$ using the 
natural $\operatorname{map} R \rightarrow R / I^{2}$.

Proof. The correspondence $P \leftrightarrow P / I^{2}$ is a one-to-one correspondence between $\operatorname{SSpec}(R)$ and $\operatorname{SSpec}\left(R / I^{2}\right)$. We use the Nöther isomorphisms to identify $R / P$ with $\left(R / I^{2}\right) /\left(P / I^{2}\right)$ and $R / P Q$ with $\left(R / I^{2}\right) /\left(P / I^{2}\right)\left(Q / I^{2}\right)$.

Finally, we define $T:$ (grass) $^{o p} \rightarrow S R n g$. Let $F \in$ grass with associated graph $G$. We first define a sequence of abelian groups $T_{i} F, i=0,1,2, \cdots$

Let $A_{G}^{0}=V_{G}$ and $A_{G}^{n}$ be the set of all sequences of arrows

$$
x_{0} \longrightarrow x_{1} \longrightarrow \cdots \longrightarrow x_{n}
$$

in $G$. Given such a sequence of arrows we may form the left $R\left(x_{0}\right)$ and right $R\left(x_{n}\right)$ bimodule

$$
M\left(x_{0}, x_{1}\right) \otimes_{R\left(x_{1}\right)} M\left(x_{1}, x_{2}\right) \otimes \cdots \otimes_{R\left(x_{n-1}\right)} M\left(x_{n-1}, x_{n}\right) .
$$

We define $T_{0} F$ to be the ring direct product $\Pi R(x), x \in V_{G}$ and $T_{n} F$, $n>0$, to be the abelian group direct product over $A_{G}^{n}$ of the above bimodules.

Now set $T F=\bigoplus_{0}^{\infty} T_{i} F$, the direct sum of abelian groups. To define a ring structure on $T F$ it is sufficient to define $p q$ for

$$
p \in M(x, \cdot) \otimes \cdots \otimes M(\cdot, y) \text { and } q \in M(z, \cdot) \otimes \cdots \otimes M(\cdot, w) \text {. }
$$

We define $p q=p \otimes q$ if $y=z$ and $p q=0$ otherwise. If $p$ or $q$ is in $T_{0} F$ we omit the tensor symbol. With this definition $T_{0} F$ is a semi-simple ring with d.c.c., $T_{1} F$ is a $T_{0} F$ bimodule and $T F$ is the tensor ring of $T_{1} F$ over $T_{0} F$.

For a split ring structure on $T F$ we take $\Gamma=T_{0} F, I=\bigoplus_{1}^{\infty} T_{i} F$ and $A=T_{1} F$. Then $T F=\Gamma \oplus A \oplus I^{2}$ as $\Gamma-\Gamma$ bimodules and we use the natural projections and injections for $(a, b, c, d)$.

Next assume that $\eta: F \rightarrow F^{\prime} \in$ grass. To define a ring homomorphism $T \eta: T F^{\prime} \rightarrow T F$ it is sufficient to construct $T_{0} \eta: T_{0} F^{\prime} \rightarrow T_{0} F$ a ring homomorphism and $T_{1} \eta: T_{1} F^{\prime} \rightarrow T_{1} F$ compatible with $T_{0} \eta$.

We define $T_{0} \eta$ to be the unique map such that all diagrams

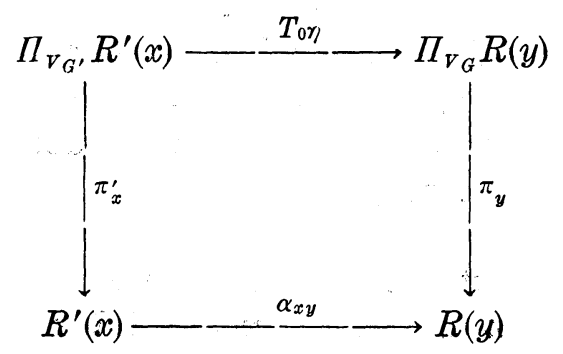


commute where the vertical maps are direct product projections and $\alpha_{x y}$ is $\eta(x)$ if $y=\eta_{G} x$ and $\alpha_{x y}=0$ otherwise. $T_{1} \eta$ is defined similarly after replacing $V_{G}$ by $A_{G}$.

\section{Properties of the functors.}

Proposition 2. (grass) $^{o p} \rightarrow S R n g$ is a functor and $S T$ is naturally equivalent to the identity functor on grass.

Proof. Let $\eta: F \rightarrow F^{\prime} \in$ grass. We must first verify that $T \eta$ is a morphism of split rings. Let $A$ be a maximal two-sided ideal of $T_{0} F$. Then there is an $x \in V_{G}$ such that $A$ is the kernel of the projection $\pi_{x}: T_{0} F \rightarrow R(x)$. Then

$$
T_{0} F^{\prime} \stackrel{T_{0 \eta}}{\longrightarrow} T_{0} F \stackrel{\pi^{\prime} x}{\longrightarrow} R(x)=T_{0} F^{\prime} \stackrel{\pi_{y}}{\longrightarrow} R^{\prime}(y) \stackrel{\eta(x)}{\longrightarrow} R(x)
$$

where $y=\eta_{G} x$. Since $R(x)$ and $R^{\prime}(y)$ are simple and $\eta(x)$ is a homomorphism of rings with unit, $\eta(x)$ is one-to-one and

$$
\operatorname{ker} \eta(x) \pi_{y}^{\prime}=\operatorname{ker} \pi_{y}^{\prime}
$$

which is a maximal two-sided ideal of $T_{0} F^{\prime}$. Thus (R4) is satisfied.

LEMma. Let $R \in S R n g$ with $I^{2}=0, \operatorname{SSpec}(R)=\left\{P_{1}, \cdots, P_{n}\right\}$ and and $\beta: \Pi_{1}^{n} R / P_{i} \rightarrow \Gamma$ the natural isomorphism. Let $e_{i}$ be the image of the identity of $R / P_{i}$ under $b \beta$. Then $e_{i} e_{j}=\delta_{i j}, 1=\sum e_{i}$ and the natural map $R \rightarrow R / P_{i} P_{j}$ carries $e_{i} I e_{j}$ monomorphically onto the Jacobson radical of $R / P_{i} P_{j}$.

Proof. Clearly $\sum e_{i}=1$ and $e_{i} e_{j}=\delta_{i j}$. Since $I=\bigcap_{1}^{n} P_{i}$ we see that $P_{i}=\sum e_{k} X_{k}$ where $X_{k}=R$ if $k \neq i$ and $X_{i}=I$. Similarly $P_{j}=$ $\sum X_{k} e_{k}$ where $X_{k}=R$ if $k \neq j$ and $X_{j}=I$. Thus we have $P_{i} P_{j}=$ $\sum e_{k} X_{k l} e_{l}$ where $X_{k l}=R$ if $k \neq i$ or $l \neq j, X_{i l}=I$ of $l \neq j, X_{k j}=I$ if $k \neq i$ and $X_{i j}=I^{2}=0$.

Now as $\Gamma$-bimodules, $R=I \oplus b \Gamma$ and hence $e_{k} I e_{l}=e_{k} R e_{l}$ if $k \neq l$. Thus writing $R=\sum e_{k} R e_{l}$ we see that if $i \neq j$ then $R / P_{i} P_{j}$ is isomorphic to the matrix ring

$$
\left[\begin{array}{cc}
e_{i} R e_{i} / e_{i} I e_{i} & e_{i} I e_{j} \\
0 & e_{j} R e_{j} / e_{j} I e_{j}
\end{array}\right]
$$

Since $\beta^{-1} a$ carries $e_{k} R e_{k}$ onto $R / P_{k}$ with kernel $e_{k} I e_{k}$ we see that the lemma holds for $i \neq j$. For $i=j$ we have that $R / P_{i} P_{j}$ is isomorphic to $e_{i} R e_{i}$ which has radical $e_{i} I e_{i}$, since $I^{2}=0$ implies that $I$ is the radical of $R$. 
Now let $R^{\prime} \in S R n g$ and set $R=T S R^{\prime}$. Identify $S R^{\prime}$ and $S\left(R^{\prime} / I^{2}\right)$ (Proposition 1). We define a map $R \rightarrow R^{\prime}$ as follows. Since

$$
\Gamma=T_{0} S R^{\prime}=\Pi_{1}^{n} R^{\prime} / P_{i}^{\prime}
$$

we take $\beta: \Gamma \rightarrow \Gamma^{\prime}$ as in the lemma. An arrow $P_{i}^{\prime} \rightarrow P_{j}^{\prime}$ is in the graph of $S R^{\prime}$ iff the radical of $R^{\prime} / P_{i}^{\prime} P_{j}^{\prime}$ is nonzero and then the associated stalk is the radical. The group $T_{1} S R^{\prime}$ is the product of these radicals. On the othe hand, $I^{\prime} / I^{\prime 2}$ is the product of the groups $e_{i}\left(I^{\prime} / I^{2}\right) e_{j}$. Thus we have a mapping $\delta: T_{1} S R^{\prime} \rightarrow R^{\prime}$ and this mapping is a $T_{0} S R^{\prime}$-bimodule isomorphism. Thus, by the remarks preceding the lemma we have a ring homomorphism $\Phi(R): T S R^{\prime} \rightarrow R^{\prime}$ which satisfies (R3). Since $\beta$ and $\delta$ are isomorphisms (R4) and (R5) are satisfied as well. It is not difficult to see that $\Phi(R)$ is natural in $R$.

THEOREM 1. The functors $S:(S R n g)^{o p} \rightarrow$ grass and $T:(\text { grass })^{o p} \rightarrow$ SRng are adjoints of the type

$$
\operatorname{Hom}(T F, R) \cong \operatorname{Hom}(S R, F) \text {. }
$$

Proof. If we consider $S$ to be a functor $S R n g \rightarrow(\text { grass })^{o p}$ then the theorem states that $S$ is a right adjoint for $T$. Let

$$
\Psi(F): F \longrightarrow S T F \in(\text { grass })^{o p}
$$

be the natural equivalence of Proposition 2. To prove the theorem it is sufficient (see [4] or [10]) to show that the compositions

$$
S[\Phi(R)] \circ \Psi(S R): S R \longrightarrow S T S R \longrightarrow S R
$$

and

$$
\Phi(T F) \circ T(\Psi(F)]: T F \longrightarrow T S T F \longrightarrow T F
$$

are identities. This is almost clear.

3. Split semi-primary rings.

Definition. A split ring $R=\Gamma+I$ is semi-primary if $I^{n}=0$ for some $n>0$.

A semi-primary split ring is semi-primary in the usual sense with Jacobson radical $I$.

In what follows we shall often confuse the quadruple $\Phi(R)$ with its first component.

Proposition 3. Let $R$ be a semi-primary split ring. Then $\Phi(R)$ : $T S R \rightarrow R$ is onto with kernel contained in $\left(\bigoplus_{1}^{\infty} T_{i} S R\right)^{2}$. 
Proof. From the discussion preceding Theorem 1 we see that $\Phi(R)$ carries $T_{0} S R$ isomorphically onto $b \Gamma$ and $T_{1} S R$ isomorphically onto $A=\operatorname{Im} d$. Thus $I=I^{2}+\phi(R)\left[\bigoplus_{1}^{\infty} T_{i} S R\right]$. Since $I$ is nilpotent it follows from [8, Lemma 1.2] that $\Phi(R)$ is onto. It follows from (R3) that the kernel is in $\left(\bigoplus_{1}^{\infty} T_{i} S R\right)^{2}$.

Definition. Let $F \in$ grass with graph $G$. We say that $F$ has no closed paths if for each path $x \rightarrow y \rightarrow z \cdots \rightarrow z$ in $G, x \neq z$.

Suppose that $F$ has no closed paths, then $T F$ is isomorphic to a generalized triangular matrix ring in Harada's sense [5]. To see this first index the vertices of the graph of $F$ as follows. First assign the integers $1,2, \cdots, n_{k_{1}}$ to those vertices which are not the tails of any arrows. Erase these vertices and all arrows leading to them obtaining a graph $G^{\prime}$. Assign the integers $n_{k_{1}}+1, \cdots, n_{k_{2}}$ to these vertices of $G^{\prime}$ which are not the tails of any arrows in $G^{\prime}$. Continue in this manner until $V_{G}$ is exhausted. Now set

$$
M_{i i}=R_{i}=R\left(x_{i}\right)
$$

and let $M_{i j}$ be the direct sum of all left $R_{i}$ and right $R_{j}$ modules $M\left(x_{i}, \cdot\right) \otimes \cdots \otimes M\left(\cdot, x_{j}\right)$ for which $x_{i} \rightarrow \cdots \rightarrow x_{j}$ is a path in $G$. The multiplication in $T F$ then induces functions $M_{i j} \times M_{j k} \rightarrow M_{i k}$ and the resulting generalized triangular matrix ring $T\left(R_{i}, M_{j k}\right)$ is isomorphic to $T F$.

Since $T_{0} F=\Gamma$ is semi-simple, $M \otimes_{\Gamma} N=0 \Rightarrow M=0$ or $N=0$. Thus we have

Proposition 4. Let $F \in$ grass then $T F$ is a semi-primary split ring iff $F$ has no closed paths.

The next statement rephrases known results in our language. By results in [1] we need not distinguish between left and right homological dimensions.

THEOREM 2. Let $R$ be a semi-primary split ring. There is an $F \in$ grass $\ni R \cong T F \in S R n g$ if and only if the ring $R$ is hereditary.

Proof. Assume that $R$ is hereditary. Since $I$ is the radical of $R$ [3, Theorem 8] shows that the global dimension of $R / I^{2}$ is finite. Thus by [6, Theorem 3.1] SR has no closed paths and hence TSR is a semi-primary split ring. Since $\Phi(R): T S R \rightarrow R$ is onto with kernel in $\left(\bigoplus_{1}^{\infty} T_{i} S R\right)^{2}$ [2, Theorem I] shows that $\Phi(R)$ is an isomorphism.

Conversely, assume $R \cong T F$. Then $T F$ is semi-primary and by [9, Proposition 4] $T F$ is an hereditary ring. 
An example due to Zaks ([11] or [12]) shows that not every hereditary generalized triangular matrix ring is split and hence not every hereditary generalized triangular matrix ring is of the form $T F$ for some $F \in$ grass.

Definition. A morphism $\eta: C \rightarrow F \in$ grass is a covering if the corresponding map on graphs, $H \rightarrow G$, is onto and for each $x \in V_{H}$ or $\alpha \in A_{H}$ the stalkwise maps $\eta(x)$ and $\eta(t a, h a)$ are identities.

LEMmA. If $F \in$ grass then there is a covering $\eta: C \rightarrow F$ in grass where $C$ has no closed paths. Further, one may assume that $C$ has at most one path between any two vertices of its graph.

Proof. Let $G$ be the graph of $F$ and let $x \in V_{G}$. We define a graph $G^{\prime}$ as follows. $V_{G^{\prime}}=V_{G} \Pi\left\{x^{\prime \prime}\right\}$ (disjoint union) and $A_{G^{\prime}}=A_{G}$. Let $h, t: A_{G} \rightarrow V_{G}$ be the head and tail functions for $G$,

$$
H_{x}=\left\{a \in V_{G} \mid h(a)=x\right\} \text { and } T_{x}=\left\{a \in V_{G} \mid t(a)=x\right\} .
$$

Choose decompositions $H_{x}=H_{1} \Perp H_{2}$ and $T_{x}=T_{1} \Perp T_{2}$.

We next define $h^{\prime}, t^{\prime}: A_{G^{\prime}} \rightarrow V_{G^{\prime}}$. If $a \in H_{x}$ we set $h^{\prime}(a)=x$ if $a \in H_{1}$ and $h^{\prime}(a)=x^{\prime}$ if $a \in H_{2}$. If $a \in A_{G^{\prime}} \backslash H_{x}$ we set $h^{\prime}(a)=h(a)$. If $a \in T_{x}$ we set $t^{\prime}(a)=\approx$ if $a \in T_{1}$ and $t^{\prime}(a)=x^{\prime}$ if $a \in T_{2}$. If $a \in A_{G^{\prime}} \backslash T_{x}$ we set $t^{\prime}(a)=t(a)$. This defines $G^{\prime}$. We shall say that $G^{\prime}$ is uotained from $G$ by making a cut at $x$.

Now define a morphism of graphs $\eta_{G}: G^{\prime} \rightarrow G$ by taking $\eta_{G}$ to be the identity on $V_{G^{\prime}} \backslash\{x\}$ and $A_{G^{\prime}}$ and setting $\eta_{G}\left(x^{\prime}\right)=x$. We extend $\eta_{G}$ to a covering $\eta: C \rightarrow F$ by "pulling the stalks back along $\eta_{G}$." That is, if $x \in V_{G^{\prime}}$ we set the stalk over $x$ equal to $R\left(\eta_{G} x\right)$, the stalk of $F$ over $\eta_{G}(x)$, and let $\eta(x): R\left(\eta_{G} x\right) \rightarrow R\left(\eta_{G} x\right)$ be the identity. Similarly for the stalks over arrows.

Since the composition of coverings is a covering we may make successively a finite number of cuts to obtain a covering $C \rightarrow F$ where $C$ has no closed paths.

In proving the second statement we may assume that $F$ has no closed paths. If $x, y \in V_{G}$ such that there are two paths from $x$ to $y$ whose final arrows $\alpha, \beta$ are such that $\alpha \neq \beta$ then make a cut at $y$ such that $\alpha$ and $\beta$ no longer have the same head. This gives $a$ covering $F_{1} \rightarrow F$. If there are two paths in the graph of $F_{1}$ with the same beginning and end, make another cut and get a covering $F_{2} \rightarrow F_{1}$, etc. This process will end in a finite number of steps.

If $\eta: C \rightarrow F$ is a covering and $C$ has no closed paths then $T \eta$ : $T F \rightarrow T C$ is a morphism of $T F$ to a split, hereditary generalized 
triangular matrix ring. Further assume that there is at most one path between any two vertices of $C$ and let $R^{\prime}$ be the set theoretic image of $T \eta$ in $T C$. The $(x, y)$ entry of the matrix ring $T C$ is zero if there is no path from $x$ to $y$ in the graph of $C$ or varies in the $R(x), R(y)$-bimodule associated to the unique path from $x$ to $y$. If the path from $x$ to $y$ has the same image under $\eta$ as the path from $z$ to $w$ then, as abelian groups, the $(x, y)$ module for $T C$ and the $(z, w)$ module for $T C$ are identical and if a matrix lies in $R^{\prime}$ then its $(x, y)$ entry equals its $(z, w)$ entry. The ring $R^{\prime}$ is obtained from $T C$ by setting certain entries of $T C$ equal to each other. For examples of rings $R^{\prime}$ see [13, Examples 5.7, 6.7, and 7.7].

Now suppose that $F=S R$ where $R$ is a semi-primary split ring. We have $\Phi(R): T F \rightarrow R$, an onto ring homomorphism. Thus $R$ will be an image of $R^{\prime}$ if $\operatorname{ker} T \eta \subset \operatorname{ker} \Phi(R)$. If $I$ is the radical of $R$ and $I^{n-1} \neq 0, I^{n}=0$ then $\operatorname{ker} \Phi(R) \supset \bigoplus_{n}^{\infty} T_{i} F$. Thus it is sufficient to have ker $T \eta \subset \bigoplus_{n}^{\infty} T_{i} F$. This means that every path of length $n$ in $F$ should be the image of a path of length $n$ in $C$ under $\eta$. To accomplish this we merely construct $C$ from $F$ by making cuts as described in the proof of the lemma and then attach as many distinct paths of appropriate length as we need to the freshly cut ends. Thus we have

THEOREM 3. Let $R$ be a semi-primary split ring. Then there is a covering $\eta: C \rightarrow S R$ where $C$ has no closed paths and at most one path between any two vertices and $\operatorname{ker} T \eta \subset \operatorname{ker} \Phi(R)$.

The generalized triangular matrix rings $T C$ are of a special type. One is given a set of simple rings $S_{i} 1 \leqq i \leqq n$ (corresponding to the vertices of the graph $C$ ) and a set of left $S_{i}$ and right $S_{j}$ bimodules, ${ }_{i} M_{j}$ (corresponding to the arrows of $C$ ).

Form a symbolic matrix $M$ with $S_{i}$ in the $i i$ th place and ${ }_{i} M_{j}$ in the $i j$ th place. Compute the formal products $M^{k}$ using the tensor product to multiply bimodules and using the identities: $M \otimes_{8} S=M$, $S \otimes_{s} M=M$. The sequence $\left[M^{k}\right]$ stabilizes in less than $n$ steps. The ring $T C$ may be defined as the generalized matrix ring for which the $i j$ th entry of a ring element varies in the $i j$ th entry of $M^{n}$.

The condition that there be at most one path between any two vertices of $C$ means that given $i$ and $j$ there is at most one sequence $i=i_{1}, i_{2}, \cdots, i_{h}=j$ such that ${ }_{i_{l}} M_{i_{l+1}}$ is nonzero for each $l$. This means that any summations necessary to form the product $M^{k}$ are automatically defined.

Call the generalized triangular matrix rings defined in this way pure tensorial triangular rings. 
The subrings $R^{1}$ of $T C$ are easily defined in terms of $M$ or $M^{n}$. If the elements on the main diagonal of $T C$ in places $i_{1}, \cdots, i_{k}$ are to be identified, replace $S_{i_{1}}, \cdots, S_{i_{k}}$ in $M$ by the same symbol, say $S_{i_{1}}$. To do this we must of course have $S_{i_{i}}, \cdots, S_{i_{h}}$ identical as abstract rings. Next replace ${ }_{i} M_{j}$ and ${ }_{k} M_{l}$ by the same symbol if the places $i$ and $k$ as well as the places $j$ and $l$ are to be identified. This process gives a new symbolic matrix $M^{\prime}$. The subring $R^{\prime}$ of $T C$ is defined by requiring that two entries of a matrix be equal whenever the corresponding entries of $\left(M^{\prime}\right)^{n}$ are equal. Call the rings $R^{\prime}$ defined in this way special subrings of pure tensorial triangular rings. For examples of such rings see [13, Examples 5.7, 6.7, and 7.7] and below.

With this notation we may restate Theorem 3 .

THEOREM 3'. Every semi-primary split ring is a homomorphic image of a special subring of a pure tensorial triangular ring. The kernel of the homomorphism is contained in the square of the radical.

ExAmpLE. We will work through the construction of $C$ and $R^{\prime}$ for a specific ring: a finite dimensional algebra $R$ over an algebraically closed field $F$ with the radical $N$ such that $R / N$ has two simple components and $N^{4}=0$.

First notice that such rings are split. By the Wedderburn principal theorem there is a semi-simple ring $\Gamma \cong R / N$ contained in $R$ such that $R=\Gamma \oplus N$, a direct sum of $\Gamma$-bimodules. The ideal $N^{2}$ is a subbimodule of $N$ and since $F$ is the center of $R$ this bimodule structure is equivalent to a left $\Gamma \otimes_{F} \Gamma^{0}$ structure. The semi-simple ring $\Gamma$ is isomorphic to a product of full matrix algebras over $F$. The well-known isomorphism $M_{n}(F) \otimes_{F} M_{m}(F)^{\circ} \cong M_{n m}(F)$ shows that $\Gamma \otimes_{F} \Gamma^{\circ}$ is semi-simple and hence that $N^{2}$ is a bimodule summand of $N$. It follows that $R$ is a split semi-primary ring.

The stalks over the vertices of $S R$ are the simple components of $\Gamma$. Denote them by $S_{i} \cong M_{n_{i}}\left(F^{\prime}\right)$. The stalks ${ }_{i} M_{j}$ over arrows are radicals of homomorphic images of $R$. Thus they are in fact left $M_{n_{i}}(F) \bigotimes_{F} M_{n_{j}}(F)^{0}$ modules and hence are semi-simple as bimodules. It follows that ${ }_{i} M_{j}$ is isomorphic to a finite direct sum of copies of $M_{n_{i}, n_{j}}(F)$, the set of $n_{i} \times n_{j}$ matrices with entries in $F$.

The tensor product of $M_{i, j}(F)$ and $M_{j, h}(F)$ over $M_{j}(F)$ is isomorphic $M_{i, k}(F)$ as left $M_{i}(F)$ and right $M_{j}(F)$ bimodules. This isomorphism carries tensor products of individual matrices to the product of the matrices.

It follows from the last paragraph that entries of $T C$ will be formal sums of matrices and the multiplication in $T C$ will be given 
by matrix multiplication plus the distributive laws. In particular if the bimodules ${ }_{i} M_{j}$ are all simple $T C$ will be isomorphic to a subring of $M_{k}(F)$ for some $k$.

Since $\Gamma$ has two components there are ten possibilities for the graph of $S R$. These reduce to seven if $R$ is assumed indecomposable $[6, \S 2]$. Suppose the vertices and arrows of the graph $G$ are given by

$$
V=\{1,2\}, \quad A=\{12,21,11\}
$$

where 12 is an arrow from 1 to 2 etc.

We wish to find a morphism of graphs $\eta: C \rightarrow G$ where $C$ has at most one path between any two vertices. It may happen that $N^{3} \neq 0$ so we also need the condition that any path of length three in $G$ is the image of a path in $C$. One choice of $C$ and $\eta$ is given by

$$
\begin{aligned}
& V_{C}=\{1, \cdots, 9\}, \\
& A_{C}=\{41,52,61,54,76,75,87,98\}
\end{aligned}
$$

with $\eta$ defined on $V_{c}$ by

$$
\eta(k)=2 \quad \text { if and only if } k=8,6,2 .
$$

The matrix $M^{\prime}$ is $9 \times 9$. On the main diagonal $M^{\prime}$ has $S_{2}$ in the 8th, 6 th and 2 nd places and $S_{1}$ elsewhere. Off the main diagonal it has ${ }_{1} M_{1}$ in places 41,54 , and $75 ; M_{2}$ in places 52,76 , and $98 ;{ }_{2} M_{1}$ in places 63 and 87 and zeros elsewhere. If $N^{\prime}$ is the radical of $R^{\prime}$ then $R \cong R / I$ where $\left(N^{\prime}\right)^{2} \supset I \supset\left(N^{\prime}\right)^{4}$.

\section{REFERENCES}

1. M. Auslander, On the dimension of modules and algebras III, Nagoya Math. J., 9 (1955), 67-77.

2. S. Eilenberg and T. Nakayama, On the dimensions of modules and algebras $V$, Nagoya Math. J., 11 (1957), 9-12.

3. S. Eilenberg, H. Nagao and T. Nakayama, On the dimensions of modules and algebras, IV, Nagoya Math. J., 10 (1956), 87-95.

4. P. Gabriel, Des categories abélienns, Bull. Soc. Math. France, 90 (1962), 323-448.

5. M. Harada, Hereditary semi-primary rings and triangular matrix rings. Nagoya Math. J., 27 (1966), 463-484.

6. G. Helzer, The skeleton of a perfect ring, J. Algebra, 17 (1971), 451-459.

7. G. Hochschild, On the structure of algebras with nonzero radical, Bull. Amer. Math. Soc., 53 (1947), 369-377.

8. J. P. Jans, On segregated rings and algebras, Nagoya Math. J., 11 (1957), 1-7.

9. J. P. Jans and T. Nakayama, On the dimension of modules and algebras VII, Nagoya Math. J., 11 (1957), 67-76.

10. S. MacLane, Categorical algebra, Bowdin College Lecture Notes-Summer 1969.

11. A. Zaks, $A$ note on semi-primary hereditary rings, Pacific J. Math., 23 (1967), 627-628.

12. , Semi-primary hereditary rings, Israel J. Math., 6 (1968), 359-362. 
13. A. Zaks, Semiprimary rings of generalized triangular type, J. Alg., 9 (1968), 5478.

Received June 11, 1971 and in revised form September 6, 1972.

UNIVERSITY OF MARYLAND 


\section{PACIFIC JOURNAL OF MATHEMATICS}

\section{EDITORS}

\section{H. SAMELSON}

Stanford University

Stanford, California 94305

C. R. Новву

University of Washington Seattle, Washington 98105

\section{J. DuGundJI}

Department of Mathematics University of Southern California Los Angeles, California 90007

RICHARD ARENS

University of California Los Angeles, California 90024

\section{ASSOCIATE EDITORS}
E. F. BECKENBACH
B. H. NeumanN
F. WOLF
K. YoSHIDA

\section{SUPPORTING INSTITUTIONS}

\author{
UNIVERSITY OF BRITISH COLUMBIA \\ CALIFORNIA INSTITUTE OF TECHNOLOGY \\ UNIVERSITY OF CALIFORNIA \\ MONTANA STATE UNIVERSITY \\ UNIVERSITY OF NEVADA \\ NEW MEXICO STATE UNIVERSITY \\ OREGON STATE UNIVERSITY \\ UNIVERSITY OF OREGON \\ OSAKA UNIVERSITY
}

\author{
UNIVERSITY OF SOUTHERN CALIFORNIA \\ STANFORD UNIVERSITY \\ UNIVERSITY OF TOKYO \\ UNIVERSITY OF UTAH \\ WASHINGTON STATE UNIVERSITY \\ UNIVERSITY OF WASHINGTON \\ $*{ }^{*}$
AMERICAN MATHEMATICAL SOCIETY
NAVAL WEAPONS CENTER
}

The Supporting Institutions listed above contribute to the cost of publication of this Journal, but they are not owners or publishers and have no responsibility for its content or policies.

Mathematical papers intended for publication in the Pacific Journal of Mathematics should be in typed form or offset-reproduced, (not dittoed), double spaced with large margins. Underline Greek letters in red, German in green, and script in blue. The first paragraph or two must be capable of being used separately as a synopsis of the entire paper. The editorial "we" must not be used in the synopsis, and items of the bibliography should not be cited there unless absolutely necessary, in which case they must be identified by author and Journal, rather than by item number. Manuscripts, in duplicate if possible, may be sent to any one of the four editors. Please classify according to the scheme of Math. Rev. Index to Vol, 39. All other communications to the editors should be addressed to the managing editor, Richard Arens, University of California, Los Angeles, California, 90024.

50 reprints are provided free for each article; additional copies may be obtained at cost in multiples of 50 .

The Pacific Journal of Mathematics is issued monthly as of January 1966. Regular subscription rate: $\$ 48.00$ a year (6 Vols., 12 issues). Special rate: $\$ 24.00$ a year to individual members of supporting institutions.

Subscriptions, orders for back numbers, and changes of address should be sent to Pacific Journal of Mathematics, 103 Highland Boulevard, Berkeley, California, 94708.

PUBLISHED BY PACIFIC JOURNAL OF MATHEMATICS, A NON-PROFIT CORPORATION

Printed at Kokusai Bunken Insatsusha (International Academic Printing Co., Ltd.), 270, 3-chome Totsuka-cho, Shinjuku-ku, Tokyo 160, Japan. 


\section{Pacific Journal of Mathematics}

\section{Vol. 44, No. $2 \quad$ June, 1973}

Tsuyoshi Andô, Closed range theorems for convex sets and linear liftings . . . . . . 393

Richard David Bourgin, Conically bounded sets in Banach spaces . . . . . . . . . 411

Robert Jay Buck, Hausdorff dimensions for compact sets in $R^{n} \ldots \ldots \ldots \ldots \ldots \ldots . \ldots 421$

Henry Cheng, A constructive Riemann mapping theorem ................ 435

David Fleming Dawson, Summability of subsequences and stretchings of

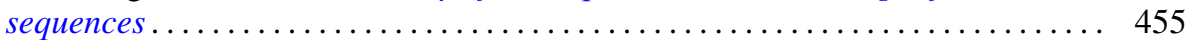

William Thomas Eaton, A two sided approximation theorem for 2-spheres ....... 461

Jay Paul Fillmore and John Herman Scheuneman, Fundamental groups of compact complete locally affine complex surfaces ....................... 487

Avner Friedman, Bounded entire solutions of elliptic equations . . . . . . . . . . . 497

Ronald Francis Gariepy, Multiplicity and the area of an $(n-1)$ continuous

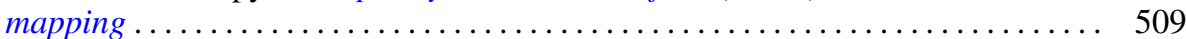

Andrew M. W. Glass, Archimedean extensions of directed interpolation groups . . . . 515

Morisuke Hasumi, Extreme points and unicity of extremum problems in $H^{1}$ on

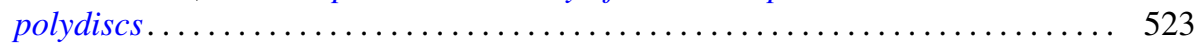

Trevor Ongley Hawkes, On the Fitting length of a soluble linear group . . . . . . 537

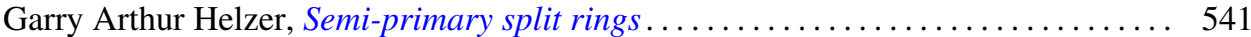

Melvin Hochster, Expanded radical ideals and semiregular ideals . . . . . . . . . 553

Keizō Kikuchi, Starlike and convex mappings in several complex variables . . . . . . 569

Charles Philip Lanski, On the relationship of a ring and the subring generated by its

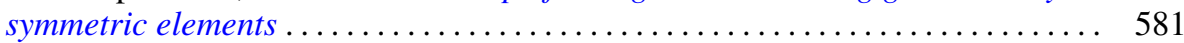

Jimmie Don Lawson, Intrinsic topologies in topological lattices and semilattices ........................................... 593

Roy Bruce Levow, Counterexamples to conjectures of Ryser and de Oliveira ...... 603

Arthur Larry Lieberman, Some representations of the automorphism group of an infinite continuous homogeneous measure algebra ..........

William George McArthur, $G_{\delta}$-diagonals and metrization theorems $\ldots .$.

James Murdoch McPherson, Wild arcs in three-space. II. An invariant of

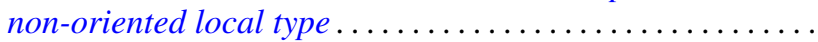

H. Millington and Maurice Sion, Inverse systems of group-valued measures ...

C. Edward Moore, Concrete semispaces and lexicographic separation of convex

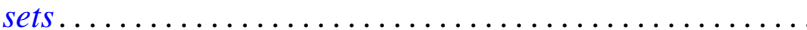

Jingyal Pak, Actions of torus $T^{n}$ on $(n+1)$-manifolds $M^{n+1}$.

Merrell Lee Patrick, Extensions of inequalities of the Laguerre and Turán type . . . . 675

Harold L. Peterson, Jr., Discontinuous characters and subgroups of finite index. . . . 683

S. P. Philipp, Abel summability of conjugate integrals . . . . . . . . . . . . . 693

R. B. Quintana and Charles R. B. Wright, On groups of exponent four satisfying an

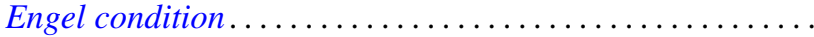

Marlon C. Rayburn, On Hausdorff compactifications. . . . . . . . . .

Martin G. Ribe, Necessary convexity conditions for the Hahn-Banach theorem in

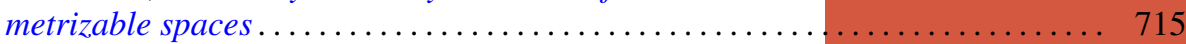

Ryōtarō Satō, On decomposition of transformations in infinite measure spaces .... 733

Peter Drummond Taylor, Subgradients of a convex function obtained from a

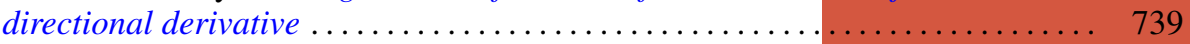

James William Thomas, A bifurcation theorem for $k$-set contractions . . . . . . . . 749 Clifford Edward Weil, A topological lemma and applications to real functions . . . . 757

Stephen Andrew Williams, A nonlinear elliptic boundary value problem . . ....... 767

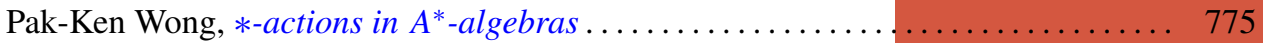

